\title{
Monitoring Ambroxol Hydrochloride Sustained-Release Tablets Release by Fiber-Optic Drug Dissolution In Situ Test System
}

\author{
Kun Nie, Li Li' ${ }^{1}$ Xinxia Li, Yan Zhang, Xuan Mu, and Jian Chen \\ School of Pharmacy, Xinjiang Medical University, No.8 Xinyi Road, Urumqi, Xinjiang 830054, \\ China
}

\begin{abstract}
The purpose of this research was to monitor in situ the drug release of ambroxol hydrochloride sustained-release tablets using a fiber-optic dissolution test system. A control experiment with a UV-vis spectrophotometer was performed simultaneously. Within $4 \mathrm{~h}$, a real-time drug release profile was obtained. The average drug release at $60 \mathrm{~min}, 120 \mathrm{~min}$, and 240 min was $23.1 \%, 52.2 \%$, and $87.6 \%$, respectively, using the fiber-optic in situ system. There were no significant differences between the two methods. The drug release of sustained-release tablets can be monitored by a fiber-optic drug dissolution in situ test system. Information about the whole dissolution process can be produced by the real-time dissolution profile.
\end{abstract}

\section{INTRODUCTION}

I he drug dissolution release test is a requirement for solid dosage form pharmaceutical products. It is an increasingly important technique within the pharmaceutical industry because it provides valuable information on batch conformity, potentially the bioavailability of the active component of the formulation, the control of process variables, and the effects of formulation changes upon the drug release. The technique is therefore routinely used for quality control purposes as well as formulation development.

Traditionally, in a drug dissolution test, samples are withdrawn from the vessels, either manually or automatically at selected times, filtered, diluted, and analyzed by UV or HPLC. Thus a dissolution release test could require a day to complete for a UV analytical method and even longer when analyzed by HPLC (1), making dissolution analysis a time-consuming and labor-intensive procedure.

From the 1990s, the traditional methods of drug dissolution testing have been changing due to the appearance of fiber-optic dissolution systems, in which a fiber-optic probe is inserted directly into each vessel to perform measurements in situ. Real-time drug dissolution release is determined in the vessels without sample removal, greatly simplifying the testing procedure (2). Josefson et al. (3) published early research in this field in 1988. Our group (4) monitored a multicomponent solid preparation by a fiber-optic sensor system in 2000. UV fiber-optic dissolution is gaining acceptance as a powerful technique in pharmaceutical industry.

${ }^{1}$ Corresponding author.
Sustained-release tablets, also called extended-release tablets, are formulated in such a manner as to make the contained medicament available over an extended period of time following ingestion (5). They involve complicated excipients and preparation technologies. Sustainedrelease tablets often need two kinds of dissolution media to simulate the human gastrointestinal $\mathrm{pH}$-acid stage and buffer stage. Without the fiber-optic dissolution test system, release amounts of sustained-release tablets are calculated at the respective time points after being withdrawn, filtered, diluted, and determined. Two separate dissolution profiles can be obtained with the fiber-optic dissolution test system software. In this paper, a drug release test of ambroxol hydrochloride sustained-release tablets was investigated. Drug release was monitored in real time by a fiber-optic dissolution test system. With the software improvements written in our laboratory, a single release profile can be obtained successfully.

\section{MATERIALS AND METHODS \\ Materials}

A fiber-optic drug dissolution in situ test system (FODT)(Figure 1) was developed by Professor Chen Jian's group of Xinjiang Medical University and Xinjiang FOCS Biotech Development Co., Ltd. It consists of a deuterium $\left(D_{2}\right)$ lamp, six fiber-optic dipping probes, and a detector with CCD (charge-coupled device). The probe tip (Figure 2) includes an incident fiber, a reflective mirror, and a detection fiber all mounted in a stainless steel cylinder. The probe path can be altered to $0.5,1.0,2.0,3.0$, or 5.0 $\mathrm{mm}$ to determine the concentrations of drugs in situ. The system can simultaneously collect UV-vis spectra (220-600 $\mathrm{nm}$ ) from six fiber channels with low noise. Data acquisition and processing were accomplished by the 
software, which was compiled by Xinjiang FOCS Biotech Development Co., Ltd. The system was validated for both the UV-vis Spectrophotometry and Dissolution Test methods of the Chinese Pharmacopeia 2005(Ch.P)(6).

A UV-visible spectrophotometer (Cintra 40, GBC Scientific Equipment Pty. Ltd., Australia) was utilized for the offline control UV determination.

Purified water was produced in the laboratory and degassed before use. Sodium chloride, sodium hydroxide, potassium dihydrogen orthophosphate, and hydrochloric acid were all analytical reagent grade and obtained from Fuchen Chemical Reagents (Tianjin, China). Ambroxol hydrochloride reference substance (Lot No.100599-200502) was obtained from the National

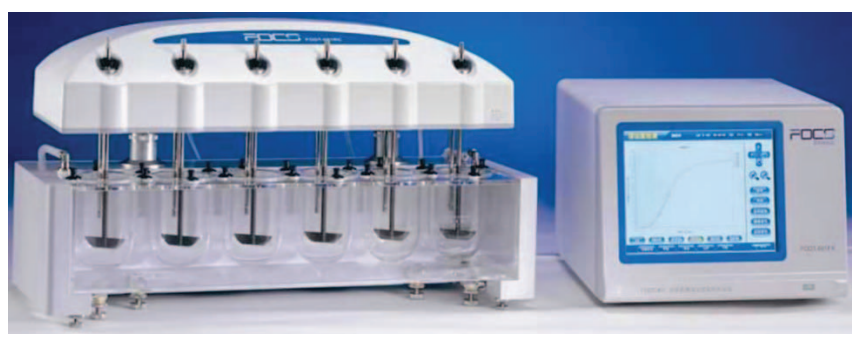

Figure 1. Fiber-optic drug dissolution in situ test system.

(A)

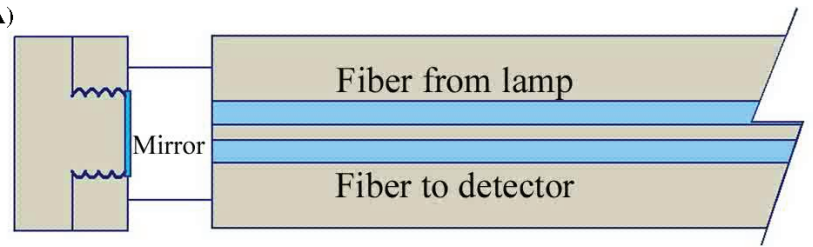

(B)

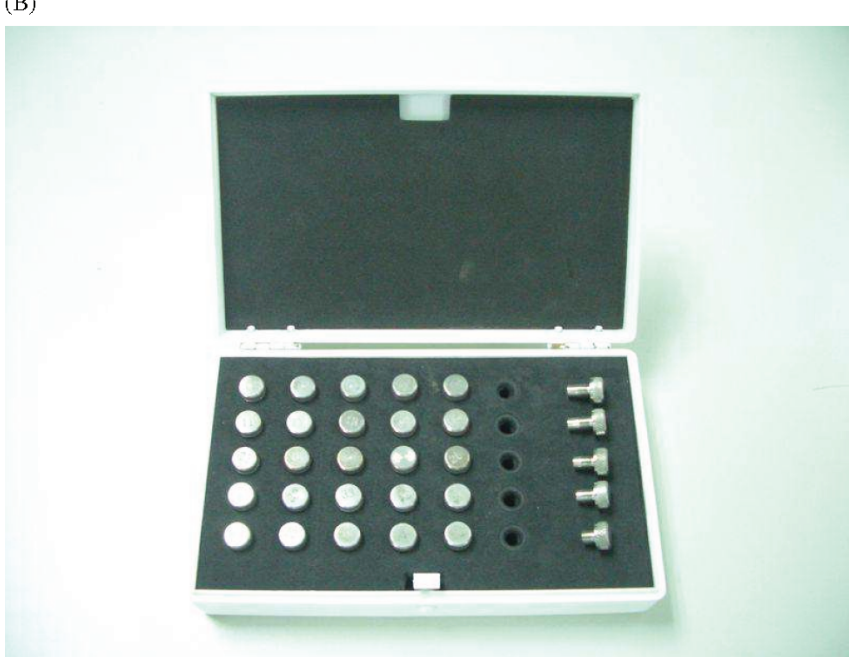

Figure 2. (A) Construction of probe and (B) photograph of five types of probes.
Institute for the Control of Pharmaceutical and Biological Products of China.

Ambroxol hydrochloride sustained-release tablets (75 mg) were supplied by Shijiazhuang Pharma.Group Zhongnuo Pharmaceutical (Shijiazhuang) Co., Ltd. (Lot No.060603).

\section{Methods \\ Preparation of Dissolution Media}

pH 1.2 Sodium Chloride and Hydrochloric Acid Solution

Sodium chloride $(2 \mathrm{~g}$ ) was dissolved in water, mixed with $7 \mathrm{~mL}$ of hydrochloric acid, and diluted to $1000 \mathrm{~mL}$ with water.

\section{pH 6.8 Phosphate Buffer}

A $0.2 \mathrm{M}$ potassium dihydrogen orthophosphate solution $(250 \mathrm{~mL})$ was mixed with $118.25 \mathrm{~mL}$ of $0.2 \mathrm{M}$ sodium hydroxide and diluted to $1000 \mathrm{~mL}$ with water.

\section{Ambroxol Hydrochloride Calibration Solutions}

Calibration solutions of ambroxol hydrochloride were prepared with $\mathrm{pH} 1.2$ and $\mathrm{pH} 6.8$ media at concentrations encompassing the expected linear absorbance range. The $\mathrm{pH} 1.2$ solutions were prepared at concentrations from 5.1 to $42.1 \mu \mathrm{g} / \mathrm{mL}$. The $\mathrm{pH} 6.8$ solutions were prepared at concentrations from 7.5 to $62.8 \mu \mathrm{g} / \mathrm{mL}$.

\section{Linearity}

The linearity of each set of standard solutions was performed to test each individual probe. Spectra and data were acquired and processed by the software.

\section{Release Test}

According to Ch.P (6), a four-hour release test was performed using Ch.P Apparatus I (same as USP Apparatus 1), in $1000 \mathrm{~mL}$ of $\mathrm{pH} 1.2$ dissolution medium for the first hour and in $1000 \mathrm{~mL}$ of $\mathrm{pH} 6.8$ phosphate buffer for the last three hours. The stirring speed was $75 \mathrm{rpm}$. The detection and reference wavelengths were set at $244 \mathrm{~nm}$ and $550 \mathrm{~nm}$, respectively. Probes with $3-\mathrm{mm}$ path lengths were used. The data acquisition interval for FODT can be chosen for any time greater than 13 seconds. The release data and profile were acquired within 4 hours.

For the UV control analysis, the sampling time points were set at $1 \mathrm{~h}, 2 \mathrm{~h}$, and $4 \mathrm{~h}$. Sample absorbances were measured at $244 \mathrm{~nm}$ with a UV-vis spectrophotometer, and the corresponding blank was measured.

\section{RESULTS}

\section{Linearity}

A proper absorbance range can be obtained with a probe path length of $3 \mathrm{~mm}$. The calibration curve equations of six channels in each of the two dissolution media are shown in Table 1.

The average correlation coefficients $(r)$ for six channels were 0.99992 with the concentration from 5.1 to 


\begin{tabular}{|c|c|c|c|c|}
\hline \multicolumn{5}{|c|}{ Table 1. Calibration Curve Equations of Six Channels } \\
\hline Channel & pH 1.2 & $r$ & pH 6.8 & $r$ \\
\hline & $A=0.0137 C+0.00421$ & 0.99996 & $A=0.0136 C+0.00244$ & 0.99988 \\
\hline & $A=0.0137 C+0.00119$ & 0.99999 & $A=0.0137 C+0.00653$ & 0.99990 \\
\hline 3 & $A=0.0136 C+0.00484$ & 0.99999 & $A=0.0137 C+0.00218$ & 0.99989 \\
\hline & $A=0.0138 C+0.00570$ & 0.99987 & $A=0.0138 C+0.00187$ & 0.99975 \\
\hline & $A=0.0139 C+0.00323$ & 0.99990 & $A=0.0140 C+0.00523$ & 0.99998 \\
\hline & $A=0.0137 C+0.00631$ & 0.99981 & $A=0.0135 C+0.01141$ & 0.99972 \\
\hline
\end{tabular}

$42.1 \mu \mathrm{g} / \mathrm{mL}$ in the $\mathrm{pH} 1.2$ solution and 0.99985 with the concentration from 7.5 to $62.8 \mu \mathrm{g} / \mathrm{mL}$ in the $\mathrm{pH} 6.8$ solution. The RSDs were $0.0073 \%$ and $0.0099 \%$ for the two solutions, respectively.

\section{Release Test}

Release data for ambroxol hydrochloride sustainedrelease tablets obtained by the FODT and the Ch.P control experiment are shown in Table 2. The average drug release values were $23.1 \%, 52.2 \%$, and $87.6 \%$ by the FODT system at the respective time points of $60 \mathrm{~min}, 120 \mathrm{~min}$, and $240 \mathrm{~min}$. There were no significant differences in the dissolution profiles between the FODT and the UV-vis specrophotometric methods.

The complete release profile is shown in Figure 3. Two separate profiles were obtained at first, due to the use of the two sequential media, one profile from 0-60 min (in $\mathrm{pH} 1.2$ medium) and the other profile from 60-240 min (in $\mathrm{pH} 6.8$ medium). The system had to be recalibrated for the second medium. But release dissolution is a whole process, and generating two separate profiles is not perfect. Almost all sustained-release drugs need to be release tested, so obtaining a complete release profile is important. After

Table 2. Comparison of Percentage FODT and UV Release for Ambroxol Hydrochloride Sustained-Release Tablets

\begin{tabular}{|c|c|c|c|c|c|c|}
\hline \multirow[b]{2}{*}{ Channel } & \multicolumn{2}{|c|}{$60 \mathrm{~min}$} & \multicolumn{2}{|c|}{$120 \mathrm{~min}$} & \multicolumn{2}{|c|}{$240 \mathrm{~min}$} \\
\hline & FODT & UV & FODT & UV & FODT & UV \\
\hline 1 & 22.1 & 21.6 & 51.4 & 50.5 & 87.2 & 85.5 \\
\hline 2 & 24.1 & 24.0 & 53.1 & 54.5 & 91.2 & 93.1 \\
\hline 3 & 22.2 & 22.5 & 53.0 & 52.7 & 89.2 & 89.9 \\
\hline 4 & 23.6 & 24.2 & 52.1 & 52.8 & 88.1 & 88.8 \\
\hline 5 & 24.3 & 25.4 & 55.3 & 55.8 & 87.5 & 90.5 \\
\hline 6 & 22.4 & 23.3 & 48.4 & 49.5 & 82.2 & 84.4 \\
\hline Average & 23.1 & 23.5 & 52.2 & 52.6 & 87.6 & 88.7 \\
\hline RSD (\%) & 0.04 & 0.06 & 0.04 & 0.04 & 0.03 & 0.04 \\
\hline
\end{tabular}

Dissolution Technologies | FEBRUARY 2009 improving the functionality of the software, we were able to acquire a complete profile for the entire release process.

\section{DISCUSSION AND CONCLUSION}

Fiber-optic dissolution testing was proven useful for pharmaceutical solid formulations. Simultaneous data registration was obtained with parallel detection. With a 244-nm detection wavelength and a probe path of $3 \mathrm{~mm}$, the FODT system produced release testing results for ambroxol hydrochloride sustained-release tablets that agree well with a UV-vis spectrophotometric method. The RSD obtained using the FODT method was comparable to the UV method. In addition, in situ monitoring and simpler operation made FODT remarkably better. With the improvement of the software, a complete release profile can be acquired.

Although UV spectrophotometry is a common method for dissolution analysis, it involves sample removal and manipulation, which can be a potential source of error. Active components might be adsorbed onto the sample filter, thereby causing a lower amount of dissolved drug. Even HPLC, which is commonly used but labor intensive and time consuming, has these shortcomings. With its ability to perform with great stability over many hours or even several days, this FODT system shows potential for applications in sustained-release preparations.

The in situ FODT system proved to be productive, useful, and labor saving and can acquire more information than conventional methods. The rapid in situ data collection capability of the system made it possible to characterize a real-time dissolution profile. The FODT system will be used widely for formulation development, bioequivalence research, and multicomponents assisted by chemometrics such as computational spectrophotometry.

\section{ACKNOWLEDGMENTS}

The authors would like to acknowledge Xinjiang FOCS Biotech Co., Ltd., China, for its contributions to this work.

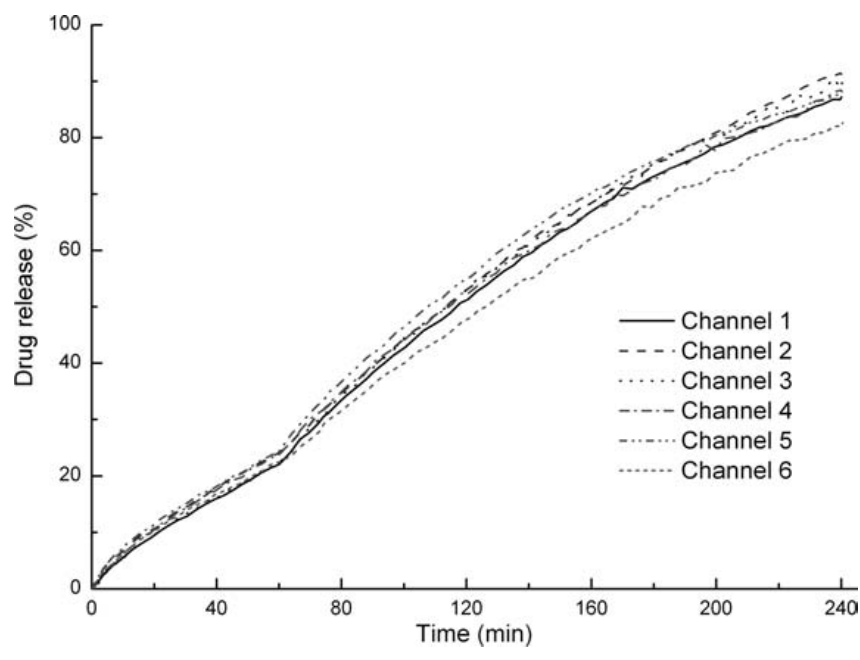

Figure 3. Release profile of ambroxol hydrochloride sustained-release tablets. 
This research was supported by the National Key Project of Scientific and Technical Supporting Programs funded by the Ministry of Science \& Technology of China during the $11^{\text {th }}$ Five-Year Plan (NO.2006BAK03A16).

\section{REFERENCES}

1. Wunderlich, M.;Way, T.; Dressman, J. B. Practical considerations when using fiber optics for dissolution testing. Dissolution Technol. 2003, 10 (4), 17-19.

2. Lu, X.; Lozano, R.; Shah, P.In-situ dissolution testing using different UV fiber optic probes and instruments. Dissolution Technol. 2003, 10 (4), 6-16.

3. Josefson, M.; Johansson, E.; Tortensson, A. Optical fiber spectrometry in turbid solutions by multivariate calibration applied to tablet dissolution testing. Anal. Chem. 1988, 60, 2666-2671.

4. Li, W.; Chen, J.; Xiang, B.; An, D. Simultaneous on-line dissolution monitoring of multicomponent solid preparations containing vitamins $\mathrm{B}_{1}, \mathrm{~B}_{2}$ and $\mathrm{B}_{6}$ by $\mathrm{a}$ fiber-optic sensor system. Anal. Chim. Acta 2000, 408, 39-47.

5. United States Pharmacopeia and National Formulary USP 30-NF 25; The United States Pharmacopeial Convention, Inc.: Rockville, MD, 2007.

6. Pharmacopoeia of the People's Republic of China; Pharmacopeia Committee of the Ministry of Public Health; Chemical Industry Press: Beijing, 2005. 